\title{
Theoretical Study of Half-Doped Models for Manganites: Fragility of the CE Phase with Disorder, Two Types of Colossal Magnetoresistances, and Charge-Ordered States for Electron-Doped Materials
}

\author{
H. Aliaga, ${ }^{1}$ D. Magnoux,${ }^{2}$ A. Moreo, ${ }^{1}$ D. Poilblanc, ${ }^{2}$ S. Yunoki, ${ }^{3}$ and E. Dagotto ${ }^{1}$ \\ ${ }^{I}$ National High Magnetic Field Lab and Department of Physics, Florida State University, Tallahassee, FL 32310. \\ ${ }^{2}$ Laboratoire de Physique Theorique CNRS-FRE2603, Université Paul Sabatier, F-31062 Toulouse, France. \\ ${ }^{3}$ International School for Advanced Studies (SISSA), via Beirut 4, 34014 Trieste, Italy.
}

(Dated: October 29, 2018)

\begin{abstract}
A comprehensive analysis of half-doped manganites is presented using Monte Carlo simulations applied to the double-exchange model with cooperative Jahn-Teller lattice distortions in two dimensions. A variety of novel results are reported. In particular: (i) The phase diagram is established in the $\lambda-J_{\mathrm{AF}}$ plane, with $\lambda$ the electron-phonon coupling and $J_{\mathrm{AF}}$ the antiferromagnetic exchange between classical $t_{2 \mathrm{~g}}$ spins. The results include standard phases, such as the CE-insulating and FM-metallic regimes, but they also include novel states, such as a ferromagnetic charge-ordered (CO) orbital-ordered phase originally predicted by Hotta et al.. This state is compatible with recent experimental results by Loudon et al. (ii) For realistic couplings, it was observed that the charge disproportionation $\delta$ of the $\mathrm{CO}$ phase is far from the widely accepted extreme limit $\delta=0.5$ of a $3+/ 4+$ charge separation. A far smaller $\delta$ appears more realistic, in agreement with recent experiments by Garcia et al. and Daoud-Aladine et al. (iii) Colossal magnetoresistance (CMR) effects are found in calculations of cluster resistances using the Landauer formalism. This occurs near the ubiquitous first-order phase transitions between the insulating and metallic states. The present result reinforces the previous conjecture that CMR phenomenology exists in two forms: the low-temperature CMR addressed here and the more standard CMR above the Curie temperature. (iv) The CE-state is found to be very sensitive to disorder since its long-range order rapidly disappears when quenched-disorder is introduced, contrary to the FM state which is more robust. This is also in qualitative agreement with recent experiments by Akahoshi et al. and Nakajima et al. (v) The phase diagram in the half-doped electron doping regime is briefly discussed as well. A charge-ordered state is found which is the analog of the $x=0.5 \mathrm{CE}$ phase. It contains a $3+/ 2+$ charge arrangement at large $\lambda$. Numerical results suggest that an approximate symmetry exists between the hole- and electron-doped systems in the large Hund coupling limit.
\end{abstract}

PACS numbers: 75.50.Pp,75.10.Lp,75.30.Hx

\section{INTRODUCTION}

Manganites are currently attracting considerable attention mainly due to the presence of the colossal magnetoresistance effect in magnetotransport measurements $\stackrel{1.2}{2}$. In addition, these materials have a complex phase diagram with a plethora of ordered phases, a typical characteristic of correlated electron systems. Many experimental and theoretical investigations have unveiled the inhomogeneous character of the states of relevance to explain the CMR phenomenon, with a competition between ferromagnetic and antiferromagnetic states that induces coexistence of clusters, typically with nanometerscale sizes ${ }^{2}$. The rationalization of this phenomenon, and concomitant explanation of the CMR effect, is based on the first-order transitions that separate the metallic and insulating phases in the clean limit (i.e. without disorder) ${ }^{1.2}$. The first-order character of the transition is caused by the different magnetic and charge orders of the competing states. The clean-limit phase diagram is illustrated in Fig. 10 (upper panel). When quenched disorder is introduced in the coupling or density that is modified to change from one phase to the other, the temperature where the Néel and Curie temperatures meet is reduced in value and eventually reaches zero as in Fig. 1 (middle panel). Upon further increase of the disorder strength, a spin disordered region appears at low temperatures, with a glassy behavior involving coexisting clusters (Fig. 10 (lower panel)). Simulations by Burgy et al $\frac{3}{n}$ have shown that the clustered state between the Curie temperature and the cleanlimit critical temperature $\left(T^{*}\right)$, with preformed ferromagnetic regions of random orientations, has a huge magnetoresistance since small fields can easily align the moments of the ferromagnetic islands, leading to a percolative conductor in agreement with experiments 4 . The quenched disorder simply triggers the stabilization of the cluster formation, but phase competition is the main driving force of the mixed state. It has been speculated ${ }^{3}$ that similar phenomena should occur in a variety of materials, including, for example, the high temperature superconductors where nanoclusters have been found ${ }^{5}$.

In spite of the phenomenological success of the previous calculations discussed above, there are many issues that must be further investigated in the manganite context to reach a reasonable understanding of these materials (for a recent discussion on open issues see Ref. (6). It is the purpose of this paper to contribute to manganite studies by addressing a variety of open topics, using computational techniques applied to realistic models for Mn-oxides. In particular, here the phase diagram at hole-density $x=0.5$ is presented for the case of cooperative Jahn-Teller phonons in interaction with carriers. Previous studies mainly focused on non-cooperative phonons ${ }^{8}$. The phase diagram reported below in Fig. 2 has interesting features. For example, it contains the realistic FM charge- 

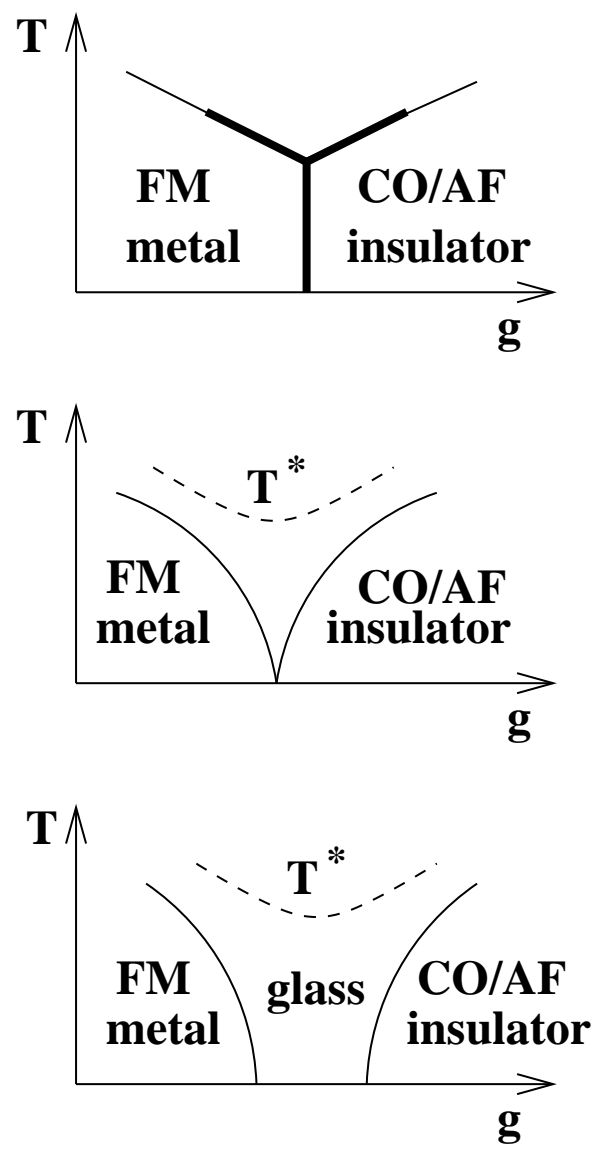

FIG. 1: Top General phase diagram of two competing phases in the absence of quenched-disorder (or when this disorder is very weak). Thick (thin) lines denote first (second) order transitions. Shown is a tricritical case, but it could be bicritical or tetracritical as well. $g$ is some parameter needed to change from one phase to the other. Middle With increasing disorder, the temperature range with first-order transitions separating the ordered states is reduced, and eventually for a fine-tuned value of the disorder the resulting phase diagram contains a quantum critical point. In this context, this should be a rare occurrence. Bottom In the limit of substantial quenched disorder, a window without any long-range order opens at low temperature between the ordered phases. This disordered state has glassy characteristics and it is composed of coexisting clusters of both phases. The size of the coexisting islands can be regulated by the disorder strength and range, and by the proximity to the original first-order transition. For more details see Refs. 23 . The new scale $T^{*}$ discussed in the text- remnant of the clean-limit transition - is also shown.

disordered and CE charge-ordered phases well-known from experiments. In addition, further evidence is provided that the transition from the FM to the AF state is first-order at low temperatures. This first-order property allows us to obtain a huge magnetoresistance effect even working on small clusters, since small magnetic fields can unbalance the ground state from antiferro to ferro, in the vicinity of the competition region. For the calculation, techniques borrowed from mesoscopic calculations using the Landauer formalism are used. The shape of the phase diagram with increasing temperature is in good qualitative agreement with recent experiments that established the bicritical form of the $x=0.5$ phase diagram 9 . Moreover, a novel phase is found with both charge and ferromagnetic order, in agreement with previous calculations ${ }^{7.8}$ and also with recent experiments by Loudon et al $\stackrel{10}{\underline{10}}$ Another feature of the results found here that is in good agreement with recent experiments (to be discussed below) is the value of the charge disproportionation. The widely accepted view of the extreme $3+/ 4+$ separation needs to be revisited. A much milder charge separation appears more realistic.

The $x=0.5$ phase diagram of the realistic model studied here is also analyzed in the presence of quenched-disorder, to test the results of phenomenological models, such as those shown in Fig. 11 Overall, good agreement with previous studies is found, but surprises are also reported such as the strong sensitivity of the CE phase to quenched disorder. This behavior is also in good agreement with recent experimental results by Akahoshi et al.$^{11}$ and Nakajima et al ${ }^{12}$, where an asymmetry between the behavior of the FM- and CE-phases was observed when disordered was introduced in some materials with $x=0.5$. Finally, since electron doping of manganites has also been experimentally actively pursued ${ }^{13}$, the model studied here is analyzed for the case of electron doping as well, with an electronic density per site equal to 1.5. A state quite similar to the CE phase is observed, which could be found in future experiments.

The overall conclusion is that theoretical studies of realistic models for manganites using unbiased techniques are unveiling a remarkable qualitative agreement with experiments. While previous phenomenological descriptions of manganites are reasonable approximations for the understanding of the puzzling CMR phenomenon ${ }^{2}$, many other features observed in the present studies still need further investigations, such as the strong sensitivity to disorder of the CE state.

\section{METHOD AND DEFINITIONS}

The Hamiltonian studied in this paper is

$$
\begin{aligned}
H & =-\sum_{\mathbf{i} \mathbf{a} \gamma \gamma^{\prime} \sigma} t_{\gamma \gamma^{\prime}}^{\mathbf{a}}\left(d_{\mathbf{i} \gamma \sigma}^{\dagger} d_{\mathbf{i}+\mathbf{a} \gamma^{\prime} \sigma}+h . c .\right)-J_{\mathrm{H}} \sum_{\mathbf{i}} \mathbf{s}_{\mathbf{i}} \cdot \mathbf{S}_{\mathbf{i}} \\
& +J_{\mathrm{AF}} \sum_{\langle\mathbf{i}, \mathbf{j}\rangle} \mathbf{S}_{\mathbf{i}} \cdot \mathbf{S}_{\mathbf{j}}+\lambda \sum_{\mathbf{i}}\left(Q_{1 \mathbf{i}} \rho_{\mathbf{i}}+Q_{2 \mathbf{i}} \tau_{\mathbf{x i}}+Q_{3 \mathbf{i}} \tau_{\mathbf{z i}}\right) \\
& +(1 / 2) \sum_{\mathbf{i}}\left(\beta Q_{1 \mathbf{i}}^{2}+Q_{2 \mathbf{i}}^{2}+Q_{3 \mathbf{i}}^{2}\right)
\end{aligned}
$$

where $d_{\mathrm{ia} \sigma}\left(d_{\mathrm{ib} \sigma}\right)$ annihilates an $e_{\mathrm{g}}$-electron with spin $\sigma$ in the $d_{x^{2}-y^{2}}\left(d_{3 z^{2}-r^{2}}\right)$ orbital at site $\mathbf{i}$, and $\mathbf{a}$ is the vector connecting nearest-neighbor (NN) sites. The first term is the NN hopping of $e_{\mathrm{g}}$ electrons with amplitude $t_{\gamma \gamma^{\prime}}^{\mathrm{a}}$ between $\gamma$ - and $\gamma^{\prime}$ orbitals along the a-direction: $t_{\mathrm{aa}}^{\mathrm{x}}=-\sqrt{3} t_{\mathrm{ab}}^{\mathrm{x}}=-\sqrt{3} t_{\mathrm{ba}}^{\mathrm{x}}=3 t_{\mathrm{bb}}^{\mathrm{x}}=t$ for $\mathbf{a}=\mathbf{x}$, and $t_{\mathrm{aa}}^{\mathbf{y}}=\sqrt{3} t_{\mathrm{ab}}^{\mathbf{y}}=\sqrt{3} t_{\mathrm{ba}}^{\mathrm{y}}=3 t_{\mathrm{bb}}^{\mathrm{y}}=t$ for $\mathbf{a}=\mathbf{y}$ (our study will be restricted to two-dimensional lattices). Hereafter, $t$ is taken as the energy unit. In the second term, the Hund coupling $J_{\mathrm{H}}(>0)$ links $e_{\mathrm{g}}$ electrons with spin $\mathbf{s}_{\mathbf{i}}=\sum_{\gamma \alpha \beta} d_{\mathbf{i} \gamma \alpha}^{\dagger} \sigma_{\alpha \beta}$ $d_{i \gamma \beta}$ ( $\sigma=$ Pauli matrices) with the localized $t_{2 \mathrm{~g}}$-spin $\mathbf{S}_{\mathbf{i}}$, assumed classical with $\left|\mathbf{S}_{\mathbf{i}}\right|=1 . J_{\mathrm{H}}$ is here considered as infinite 
or very large. The third term is the AFM coupling $J_{\mathrm{AF}}$ between NN $t_{2 \mathrm{~g}}$ spins. The fourth term couples $e_{\mathrm{g}}$ electrons and $\mathrm{MnO}_{6}$ octahedra distortions, $\lambda$ is a dimensionless coupling constant, $Q_{1 \mathbf{i}}$ is the breathing-mode distortion, $Q_{2 \mathbf{i}}$ and $Q_{3 \mathbf{i}}$ are, respectively, $\left(x^{2}-y^{2}\right)$ - and $\left(3 z^{2}-r^{2}\right)$-type JT-mode distortions, $\rho_{\mathbf{i}}=\sum_{\gamma, \sigma} d_{\mathbf{i} \gamma \sigma}^{\dagger} d_{\mathbf{i} \gamma \sigma}, \tau_{\mathbf{x i}}=\sum_{\sigma}\left(d_{\mathbf{i} a \sigma}^{\dagger} d_{\mathbf{i b} \sigma}+d_{\mathbf{i b} \sigma}^{\dagger} d_{\mathbf{i a} \sigma}\right)$, and $\tau_{\mathbf{z i}}=\sum_{\sigma}\left(d_{\mathbf{i} a \sigma}^{\dagger} d_{\mathbf{i a} \sigma}-d_{\mathbf{i b} \sigma}^{\dagger} d_{\mathbf{i b} \sigma}\right)$. The fifth term is the usual quadratic potential for adiabatic distortions and $\beta$ is the spring-constants ratio for breathing- and JT-modes. In actual manganites, $\beta \approx 2$ (see Ref. 14), and this is the value, unless something different is stated, we will consider throughout this paper. In undoped manganites, all oxygens are shared by adjacent $\mathrm{MnO}_{6}$ octahedra and the distortions are not independent, suggesting that the cooperative effects are very important. This observation is likely valid even at finite hole densities, since experiments show the presence of orbital ordering in the half-doped regime. To consider this cooperation, here oxygen ion displacements, denoted by $u_{\mathbf{i}}^{x}$ and $u_{\mathbf{i}}^{y}$, are directly optimized $^{15}$.

It has been clarified in previous literature (e.g., Ref. 2) that a large Hund coupling as used here suppresses double occupancy of the same orbital, and in this respect behaves as a Hubbard $U$ interaction. In addition, a robust coupling $\lambda$ also acts as a $U^{\prime}$ repulsion between electrons in the same site but different orbitals. As a consequence, it is a reasonable approximation to neglect the Coulombic interactions in the problem, which simplifies enormously the computational effort.

The model will be analyzed primarily using a classical Monte Carlo (MC) procedure for the localized spins and phonons, in conjunction with exact diagonalization of the conduction electron system. This last part of the process corresponds to the solution of the single-electron problem with hoppings determined by the localized spin configuration. The resulting energy levels are then filled with the number of electrons to be studied, namely the simulations are carried out in the canonical ensemble (see Appendix). However, simulations directly in the grand canonical ensemble varying chemical potentials were also carried out. In the present work, all the calculations were made on a 2D $4 \times 4$ cluster, mainly with filling $x=1 / 2$, as explained in the introduction. Currently, it is not possible to comprehensively study larger systems, unless in special cases, due to the considerable CPU time that the diagonalization process needs, and the large number of parameters that must be varied to fully explore the phase diagram. In spite of the size limitation of our effort, the results reported here have a clear physical interpretation and size effects appear to be mild for the quantities that were investigated.

Updates of the spin and phononic $\left\{\theta_{\mathbf{i}}, \phi_{\mathbf{i}}, u_{\mathbf{i}}^{x}, u_{\mathbf{i}}^{y}\right\}$ configurations are accepted or rejected according to the Metropolis algorithm. The number of MC steps per site is typically taken as 3000 for thermalization, with an additional 10000 for measurements. The simulations usually started with random states at high temperature, and then the temperature was decreased slowly, but at very low temperatures some of the simulations started with ordered states to speed up the convergence. The results of the simulations were analyzed using three separate but related quantities: the spin structure factor

$$
S(\mathbf{k})=\sum_{\mathbf{i}, \mathbf{j}}\left\langle\mathbf{S}_{\mathbf{i}} \cdot \mathbf{S}_{\mathbf{j}}\right\rangle \mathbf{e}^{\mathbf{i k} \cdot\left(\mathbf{r}_{\mathbf{i}}-\mathbf{r}_{\mathbf{j}}\right)},
$$

of the classical spins, the charge structure factor

$$
N(\mathbf{k})=\sum_{\mathbf{i}, \mathbf{j}}\left\langle\rho_{\mathbf{i}} \rho_{\mathbf{j}}\right\rangle \mathbf{e}^{\mathbf{i k} \cdot\left(\mathbf{r}_{\mathbf{i}}-\mathbf{r}_{\mathbf{j}}\right)}
$$

and the orbital structure factor defined as,

$$
T(\mathbf{k})=\sum_{\mathbf{i}, \mathbf{j}}\left\langle\mathbf{T}_{\mathbf{i}} \cdot \mathbf{T}_{\mathbf{j}}\right\rangle \mathbf{e}^{\mathbf{i k} \cdot\left(\mathbf{r}_{\mathbf{i}}-\mathbf{r}_{\mathbf{j}}\right)},
$$

with $\mathbf{T}_{\mathbf{i}}=\left(\tau_{\mathbf{x i}}, \tau_{\mathbf{y} \mathbf{i}}, \tau_{\mathrm{zi}}\right)$ and $\tau_{\mathbf{y} \mathbf{i}}=i \sum_{\sigma}\left(d_{\mathbf{i a} \sigma}^{\dagger} d_{\mathbf{i b} \sigma}-d_{\mathbf{i b} \sigma}^{\dagger} d_{\mathbf{i a} \sigma}\right)$. Frequently, visual investigations of snapshots of the spin and phononic configurations at low temperatures were useful to guide the intuition. Some dynamical properties were also studied, such as the density-of-states. For details see Ref. 2. More sophisticated techniques used to study the conductance of clusters are described later in the paper.

\section{LOW-TEMPERATURE PHASE DIAGRAM}

The phase diagram of the double-exchange model with cooperative JT phonons at $x=0.5$ is shown in Fig. 2 The properties of each phase were analyzed through the spin, charge, and orbital correlations in real and momentum space defined in the previous section. Varying $J_{\mathrm{AF}}$ three spin regimes were identified, similar to those reported in previous investigations 16 . Typical results for the evolution of the energy with $J_{\mathrm{AF}}$ are presented in Fig. 3] clearly showing the three states that compete at fixed $\lambda$. Using this procedure, the phase diagram was constructed. Note the clear first-order transitions separating the different phases, shown in the figure as level crossings.

In agreement with well-known experimental results for half-doped real manganites, a CE phase is stabilized in the intermediate $J_{\mathrm{AF}}$ range. The actual correlations for this phase will not be shown explicitly here since they have appeared in some previous investigations 16 and our results agree very well with those results. However, there are issues to remark not discussed before. For example, note that the CE phase is present not only at large $\lambda$ but also at small electron-phonon coupling. This last result suggests that a nonzero $J_{\mathrm{AF}}$ is sufficient to stabilize the zigzag chains characteristic of the $\mathrm{CE}$ region. Hotta et al .17 proposed that a band-insulator picture is needed to understand this phenomenon, and the present results support this view. It is the geometry of the zigzag chains that plays the key role in stabilizing the CE state. This result may have important consequences for experiments as discussed below. The dotted line represents a continuous transition between the standard CE phase and a novel canted CE' phase. To understand the characteristics of the new CE' state, note that the standard $\mathrm{CE}$ phase can also be interpreted as an arrangement of parallel AF zig-zag chains, displaced one lattice parameter in the $\mathrm{x}$ - and $y$-directions simultaneously. When the relative spin angle between the neighboring AF zig-zags is $\pi$, we have the normal 


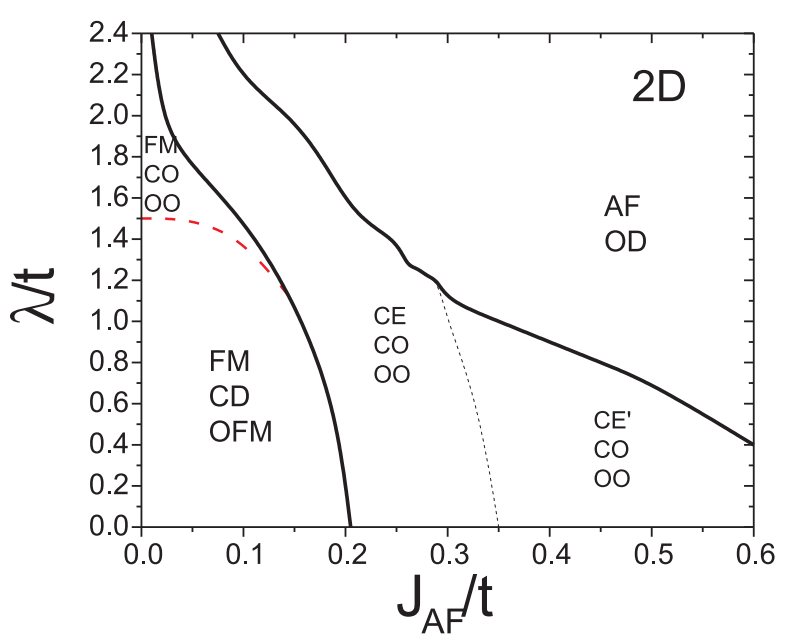

FIG. 2: Phase diagram of the half-doped 2D two-orbitals model with cooperative Jahn-Teller phonons at $T / t \sim 0$. The Hund coupling $J_{H}$ is infinite. The phases were obtained analyzing the crossing of energies (as explained in Fig. 3, and also $S(\mathbf{k}), T(\mathbf{k})$, and $N(\mathbf{k})$ (see definitions in text). The magnetic phases present are FM, CE, CE' and AF. The symbols $\mathrm{CO}(\mathrm{CD})$ stand for charge-ordered (-disordered) phases. OFM corresponds to a homogeneous state with dominant uniform $x^{2}-y^{2}$ orbital order. The staggered orbital-ordered states, OO, have the standard "CE" pattern, present in the three phases. The FM-CD-OFM phase is metallic, the rest of the phases are insulating. Thick lines denote first-order transitions, the dash-line is second order. The lines are smooth interpolations between a finite but large number of points obtained numerically.

$\mathrm{CE}$ phase. In the CE' regime this relative angle varies continuosly as $\lambda$ or $J_{\mathrm{AF}}$ are increased. Finally, when this spin angle between neighboring AF zig-zag chains is 0 , we recover the normal AF phase.

At sufficiently large $J_{\mathrm{AF}}$ coupling, an antiferromagnetic spin arrangement is found. This phase has not been observed yet experimentally, but there is no reason to believe that the state cannot exist in some Mn oxide. Within the accuracy of our study, this AF phase is believed to have no long-range order in the orbital and charge degrees of freedom. This corresponds to a nearly uniform arrangement of charge at small $\lambda$. However, at large electron-phonon coupling the situation changes qualitatively. In this regime, a "polaronic" state seems to be stabilized, where the 8 electrons present in the 16sites cluster studied here become trapped in random locations. If in this random distribution some of the electrons are close to one another, local orbital order similar to that of the $x=0$ limit was observed. Again, this AF phase has not been observed experimentally yet, but the recent progress in the discovery of new phases (see next section) suggests that this remains a serious possibility.

The regime of small $J_{\mathrm{AF}}$ coupling is dominated by FM states. The large- $\lambda$ zone has a novel state which is discussed in the next section. At intermediate and small couplings, the state that dominates at low temperature has the characteristics of the FM metallic state well-known to exist in three-dimensional Mn oxides. For instance, in the charge

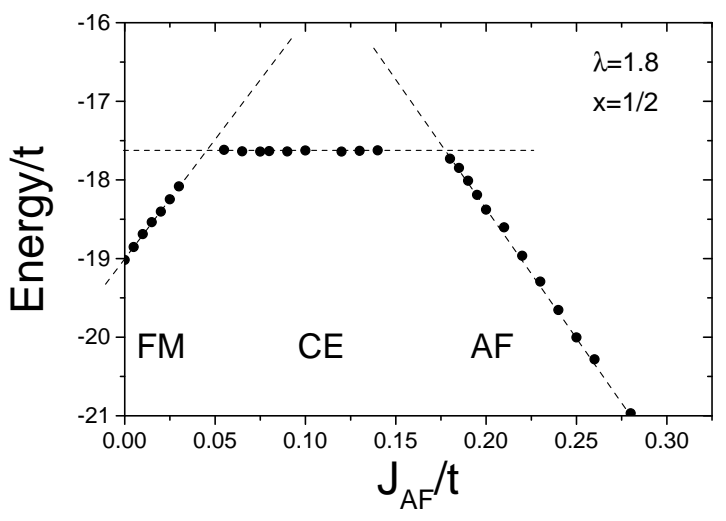

FIG. 3: MC energy vs. $J_{\mathrm{AF}}$ for $x=1 / 2, \lambda=1.8, T / t \sim 0$. This low$T$ energy is calculated after starting with a random high- $T$ state and lowering $T$ slowly. For this value of $\lambda$, the three FM, CE and AF phases have $\mathrm{OO}$ with the $d_{3 x^{2}-r^{2}}-d_{x^{2}-y^{2}}-d_{3 y^{2}-r^{2}}$ usual alternation of orbitals, distributed in zig-zags. The $x^{2}-y^{2}$ orbitals at the " $\mathrm{Mn}^{4+"}$ sites are populated at small and moderate values of $\lambda$. The three phases also have checkerboard-type charge ordering. Near the crossings of levels we have observed a notorious slowing down in the convergence of the MC calculations. Lines are guides to the eye. Very similar results were obtained at several values of $\lambda$ and were used to construct Fig. 2

sector there is no order. Regarding orbital order, the correlations suggest the presence of a uniform arrangement. This uniform orbital order at small $\lambda$ is not produced through a spontaneous-symmetry-breaking process, but it exists as a direct consequence of the form of the kinetic energy in our twodimensional simulations. To show this, the procedure is the following. Consider the limit of Hund coupling infinite as in our studies, and also $\lambda=J_{\mathrm{AF}}=0$. In this case, the Hamiltonian can be diagonalized exactly in momentum space and the ground state for the $4 \times 4$ cluster can be exactly constructed. In this state, the mean value of the number operator for the two orbitals of relevance can be calculated as well. The results are not equal for the two orbitals, but there is an asymmetry in favor of the $x^{2}-y^{2}$ orbital. Then, in this respect the uniform orbital-order in the state is explicit in the model, and it is not induced by the JT phonons. These conclusion were also checked on larger lattices, such as $20 \times 20$, and in threedimensional clusters.

\section{NOVEL FM/CO PHASE}

It is interesting to remark that the phase diagram in Fig. 2 contains not only the FM-metallic and CE-insulating phases - well-established experimentally - but other phases as well. For example, at large $J_{\mathrm{AF}}$ an $\mathrm{AF}$ phase exists in a wide range, and this phase could be observed in future experimental investigations, as already discussed. Even more exciting is the case of the ferromagnetic charge-ordered (FM/CO) phase in the 
upper-left corner of Fig. 2] This phase was found in previous investigations by Hotta et $a l \underline{\underline{7}}$ using cooperative phonons (and suggested by Yunoki et $a l^{\underline{\underline{8}}}$ as well, using non-cooperative phonons). It is quite remarkable that recent experimental efforts by Loudon et al $\stackrel{10}{!}$ have unveiled the presence of a FM insulating phase at half doping, compatible with the FM/CO state found in the Monte Carlo simulations. For completeness, in Fig. 4 the orbital arrangement of the FM/CO phase found in our simulations is shown, although it has been already presented in $\operatorname{Ref} 7$. The orbital order corresponds to the same pattern of $3 x^{2}-r^{2} / 3 y^{2}-r^{2}$ orbitals of the CE-phase, but the spins are arranged ferromagnetically, since $J_{\mathrm{AF}}$ is not large enough to induce the $\mathrm{CE}$ order. Future experiments will clarify if indeed the theoretically predicted $\mathrm{FM} / \mathrm{CO}$ regime ${ }^{7.8}$ corresponds to the results reported in Ref. 10. Note that the discovery of more phases than previously known is a recent interesting trend, both in experiments and theory. In fact, at $x=0$ a novel E-phase has been observed in simulations by Hotta $e t$ $a l \stackrel{18}{ }$, as well as in experiments by Kimura et al ${ }^{19}$. Many surprises may still be found in manganite investigations, even at the rather elementary level of characterization of states, since its strongly correlated character creates a plethora of phases that are in strong competition.

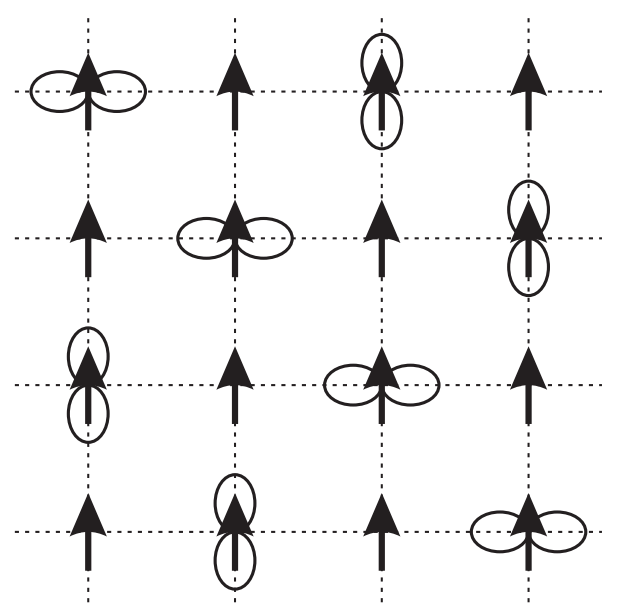

FIG. 4: Orbital, spin, and charge arrangement for the FM-CO-OO phase. The $3 x^{2}-r^{2}$ and $3 y^{2}-r^{2}$ orbitals represent the $\mathrm{Mn}^{3+}$ ions, while the empty sites represents the $\mathrm{Mn}^{4+}$ sites. This state was presented for the first time in Ref. 7. Evidence of a FM phase with checkerboard $\mathrm{CO}$ pattern has been found recently: ${ }^{10}$

\section{PHASE DIAGRAM VARYING TEMPERATURE}

The properties of the model studied here were also investigated at finite temperatures. In this case, the errors in the estimations of the phase boundaries are larger than at zero temperature. The reason is that at $T=0$, the first-order transitions can clearly be established through level crossings, which exist even in small systems. However, the continuous transitions that are found at finite $T$ can only be roughly located on small systems based on spin, charge, and orbital correlations at the largest distance available in the clusters studied. In spite of this problem, the results shown here are sufficiently accurate to understand the main trends in the phase diagrams.

Typical results can be found in Fig. 5 The level-crossing procedure shown in (a) establishes easily the phase diagram at low $T$. At finite $T$, the momentum space correlations show a rapid increase upon cooling at characteristic temperatures (see Figs. [5] b,c,d) as typical examples) due to the increase of the real-space correlations at the largest available distances. These temperatures are the best approximations to the true bulk-limit critical temperatures that our investigations can produce at present.
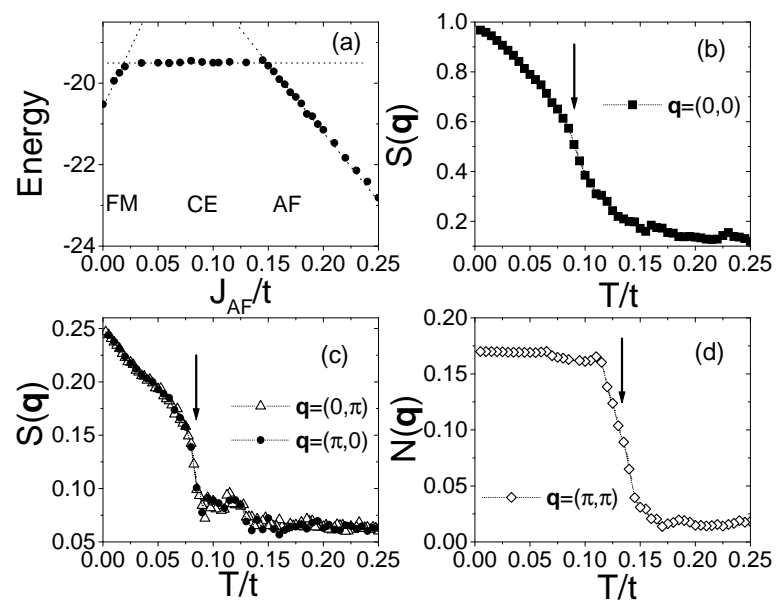

FIG. 5: (a) MC energy vs. $J_{\mathrm{AF}}$ for $x=1 / 2, \lambda=2.0, T / t \sim 0$. The location of the level crossings were used in the low- $T$ phase diagram of Fig.2] (b) Spin structure factor $\mathrm{S}(\mathbf{q})$ as a function of $T / t$, for $\mathbf{q}=(0,0)$ and $J_{\mathrm{AF}} / t=0$, indicating that at low T the FM phase is stabilized. (c) $\mathrm{S}(\mathbf{q})$ vs. $T / t$, for $J_{\mathrm{AF}} / t=0.05, \lambda=2.0$, and momenta $\mathbf{q}=(0, \pi)$ and $\mathbf{q}=(\pi, 0)$, characteristic of the CE phase. (d) Charge structure factor $\mathrm{N}(\mathbf{q})$ vs. $T / t$ for the same parameters as in (c). In (b), (c), and (d) the approximate temperatures where correlations become robust are indicated by arrows.

An example of the phase diagrams constructed by this procedure is given in Fig. 6 where the results at $\lambda=2.0$ are shown. Here, the 'oscillations' in the characteristic temperatures are indicative of the errors in our procedure, and of the clear tendency in simulations at large $\lambda$ to spend considerable Monte Carlo time trapped in competing states, as it occurs in glassy systems. In spite of these complications, the phase diagrams such as Fig. 6 are sufficiently informative to unveil the dominant properties of the system.

Some of the properties of Fig. 6 are worth explicitly discussing: (i) The Néel critical temperature of the CE state is the lowest among the three dominant states. This may explain in part its sensitivity to disorder discussed later in this paper. (ii) At large $\lambda$, charge order in the CE phase occurs at a temperature larger than the Néel temperature, as it occurs in many manganite experiments. (iii) In the range of $J_{\mathrm{AF}}$ between 0.02 and 0.03 , the CE phase is stable at low- $T$, but upon heating the FM state is stabilized, before the system becomes paramagnetic. This curious behavior is compatible with experimental results recently reported by Tomioka and Tokura ${ }^{2}$, 


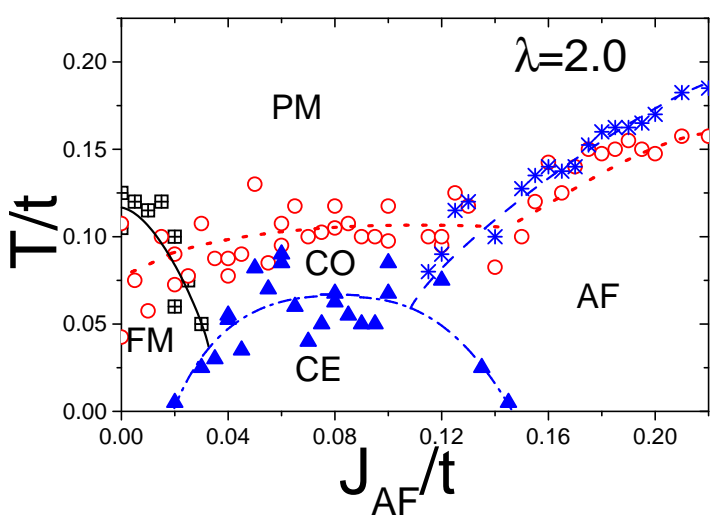

FIG. 6: Phase diagram $T / t$ vs. $J_{\mathrm{AF}} / t$ of the $2 \mathrm{D}$ two-orbitals cooperative-phonon model at $\lambda=2.0 . T_{\mathrm{C}}, T_{\mathrm{CE}}$, and $T_{\mathrm{AF}}$ are represented by squares, triangles, and asterisks, respectively. These temperatures are a crude estimation of the magnetic ordering temperatures of the FM, CE and AF phases, obtained in our small cluster simulations, as explained in the text. The oscillations in the results are indicative of the error bars in the critical temperatures. The temperatures $\left(T_{\mathrm{CO}}\right)$ where charge and orbital correlations become important upon cooling are marked by circles and a dotted line. Transitions to the paramagnetic (PM) and $\mathrm{CO}$ phases are second order. Transitions FM-CE, and CE-AF are first-order at low temperatures.

reproduced in Fig. 7 It is remarkable that the model studied here is able to qualitatively reproduce even this fine detail of the real phase diagram of $x=0.5$ manganites. The different dimensionalities between experiments and the simulations reported here are not a problem, since it is well-known that the phase diagrams of the double-exchange model are qualitatively similar in the dimensions of interest ${ }^{2}$.

In Fig. 8 the numerically obtained phase diagram at $\lambda=1.3$ is also shown, as representative of the intermediate $\lambda$ range. As in the previous cases, the information was obtained on a 16-sites lattice, using spin correlations at the largest available distances to decide which tendency dominates in the ground state. Instead of showing explicitly the numbers defining the lines as done in Fig. 6, here a smooth average is presented for simplicity. Error bars similar to those of Fig. 6 should be assumed at $\lambda=1.3$ as well. In good agreement with the results at other $\lambda \mathrm{s}$, the Curie temperature in Fig. 8 is found to be larger than the CE critical temperature, and there is a region of couplings where ferromagnetism is stabilized above the CE state, before turning paramagnetic upon further heating. The gray region denotes a regime where charge-ordering appears, but not spin order (however, note that this region with phase competition is particularly complicated to study due to the presence of many competing minima in the free energy).

Figure 9 illustrates the presence of gap and pseudogap (PG) features in the density-of-states (DOS), due to the robust value of $\lambda$ used. At low temperatures in the CE phase, there is clearly a gap due to charge ordering. However, the existence of a reduced DOS at the chemical potential in the form of a pseudogap survives the increase of the temperature, and above

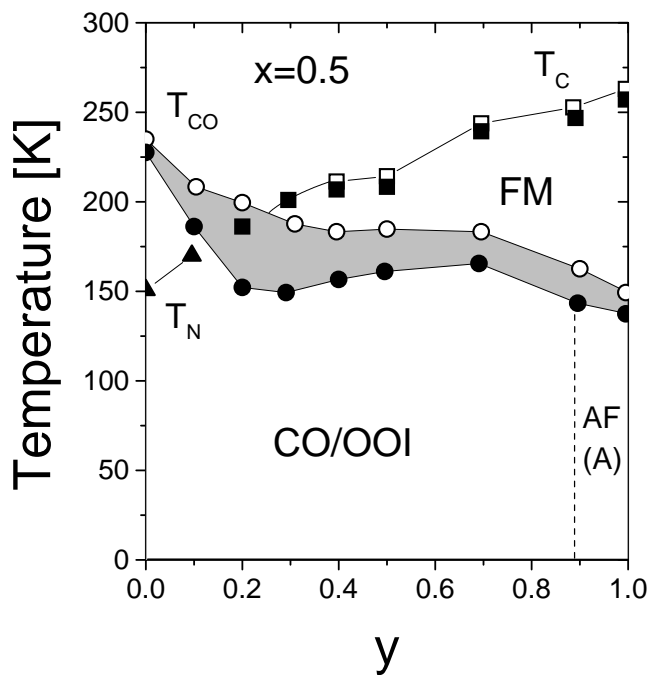

FIG. 7: Experimental phase diagram of the half-doped compound $\operatorname{Pr}_{0.5}\left(\mathrm{Ca}_{1-y} \mathrm{Sr}_{y}\right)_{0.5} \mathrm{MnO}_{3}$, reproduced from Ref 9 . The chargeand orbital-ordered insulator and ferromagnetic metallic states are denoted by CO-OOI and FM, respectively. The transitions from (to) the CO-OOI phase are represented by circles. The transitions to the FM phase are represented by squares. The Néel temperature is represented by solid triangles. The grey area indicates hysteresis. Note the presence of a FM phase above a $\mathrm{CO} / \mathrm{AF}$ phase, compatible with our results, as discussed in the text.

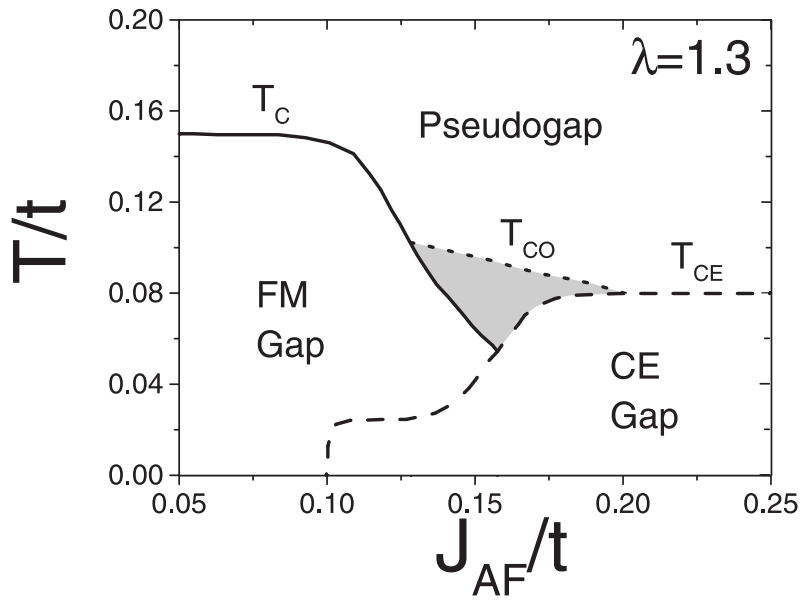

FIG. 8: Phase diagram of the two-orbitals model with cooperative Jahn-Teller phonons at $\lambda=1.3$ and $\beta=100$. The results are smooth interpolations using the numerically available data, and in this respect they are not exact. The notation is standard. Note that at this coupling there are pseudogap features in the density-of-states above the ordering temperatures, and a hard gap below those critical temperatures. The gray area is a region where the simulations give a mixture of orders, for the large number of iterations carried out.

the Néel temperature the effect appears to be caused by dynamical Jahn-Teller distortions. This result reinforces the notion that a large depletion of the DOS at the chemical potential should be present in a broad range of couplings and temperatures in models for manganites. The existence of a PG feature 


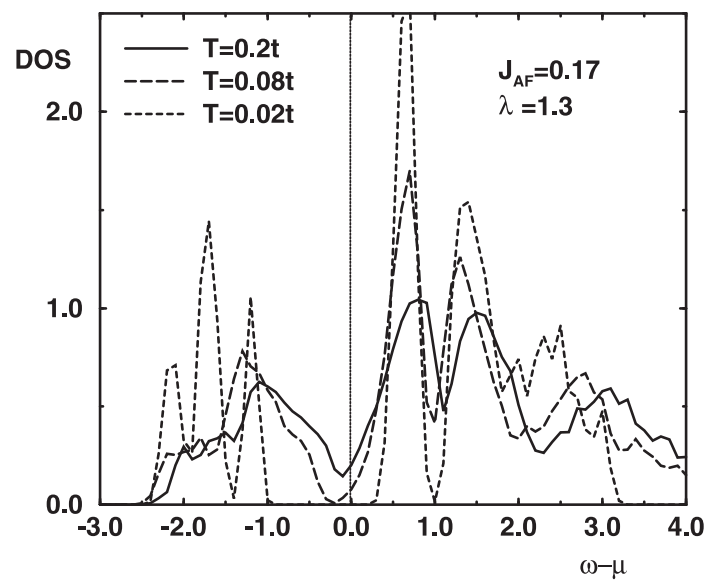

FIG. 9: Density-of-states obtained numerically at the couplings and temperatures indicated. Comparing with Fig. 8 these results show that pseudogap features appear in the DOS above the ordering Néel temperature of the CE state, at $\lambda=1.3$ and $\beta=100$. Pseudogaps have also been observed in many previous investigations (see Refs. 20 21, 22, for instance).

was first noticed theoretically by Moreo et al. 20 and experimentally in photoemission experiments by Dessau et al $\stackrel{21}{\underline{\underline{2}}}$. More recently, in optical conductivity experiments by Noh and collaborators ${ }^{22}$, PG features were also observed. Pseudogaps in DOS alter substantially the transport properties of the system, and in addition they suggest interesting similarities with other materials -such as the cuprates- where pseudogap features have also been identified. In fact, most of the phenomenology of the $\mathrm{Mn}$ - and $\mathrm{Cu}$-oxides is similar, with phase competition being an important ingredient to understand phenomenologically their properties $\stackrel{23}{3}$.

\section{CHARGE DISPROPORTIONATION}

Our calculations allow us to address theoretically a recent controversy in the experimental literature related with the actual value of the charge disproportionation in charge-ordered half-doped manganites. The "standard folklore" since the 1950s says that the CE state is made out of a checkerboard distribution of $3+$ and $4+$ charges. However, on theoretical grounds this state appears to be too "extreme". Since the work of Yunoki and collaborators ${ }^{8}$ establishing the phase diagram of manganite models with JT phonons at $x=0.5$, it has been noticed that the $\mathrm{CE}$ phase is close to a ferromagnetic metallic phase only at small and intermediate values of the electron-phonon coupling $\lambda$. Having the two phases close to one another is important to understand the phenomenology of $\mathrm{La}_{1-x} \mathrm{Ca}_{x} \mathrm{MnO}_{3}$ (LCMO), where it is known that the two phases "touch" at $x=0.5$, in the phase diagram varying the $\mathrm{Ca}$ concentration. If indeed $\lambda$ is not very large, then the chargeordered state should be far from the 3+/4+ extreme separation. In fact, recent experimental work by Garcia et al., DaoudAladine $e t$ al., and others have revealed a very weak signal for charge disproportionation, and the standard $3+/ 4+$ has been severely questioned 24 .

How can we understand this challenging new phenomenology? A recent key observation by Hotta et al $\stackrel{17}{17}$ indicates that the CE state may have an origin totally different from the "large Coulomb repulsion" view of Goodenough (or the large $\lambda$ analog in the presence of JT distortions). In the new perspective, the formation of the zigzag chains of the CE state can occur even at very small $\lambda$ since these chains lead to the optimization of the kinetic energy in the presence of a nonzero $J_{\mathrm{AF}}$. In this context, the insulating character arises mainly from a band-insulator picture, already present in the study of individual zigzag chains. The validity of this view is clear in the phase diagram of Fig. 2 that shows a CE phase that $e x$ tends to $\lambda=0$, a result quite difficult to understand from the large- $\lambda$ standard perspective. Since the FM metallic and CE phases are not in contact at large $\lambda$ in the theoretical phase diagram, and since experimentally they are known to be close in energy (at least for LCMO), then the $3+/ 4+$ view of the CE state must indeed be revisited.

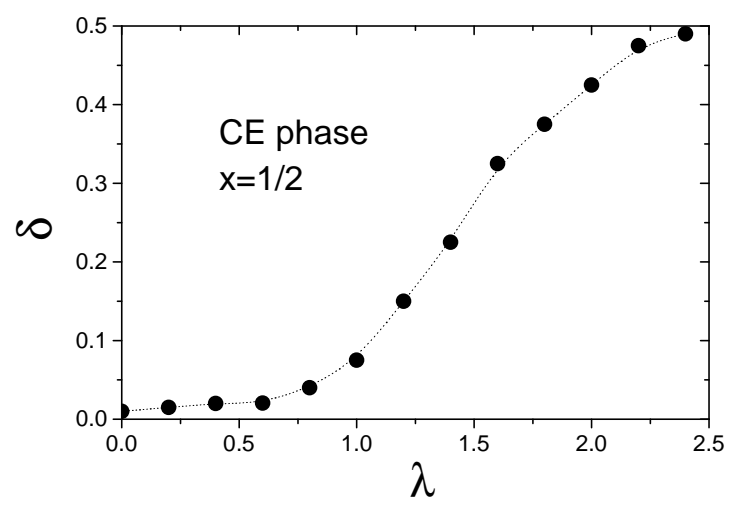

FIG. 10: Charge disproportionation $\delta$ vs. $\lambda$, calculated in the CE phase of Fig. 2 Starting at $\lambda=0$, the values of $J_{\mathrm{AF}}$ used are 0.25 , $0.25,0.25,0.25,0.2,0.2,0.2,0.15,0.15,0.1,0.1,0.05$, and 0.05 , due to the tilted shape of the CE phase. A value $\delta \approx 0.5$ corresponds to the standard charge ordering, with $\mathrm{Mn}^{3+}$ and $\mathrm{Mn}^{4+}$ arranged in a checkerboard pattern. To reproduce this extreme charge-ordered state an abnormally large value of $\lambda$ is needed. More realistic parameters correspond to a far less dramatic charge separation.

Figure 10 shows the numerically obtained charge disproportionation $\delta$ along the $\mathrm{CE}$ phase varying $\lambda$ (note that the bended nature of the CE phase in the phase diagram Fig. 2 forces us to adjust $J_{\mathrm{AF}}$ as $\lambda$ changes). The entire CE region has a nonzero $\delta$ according to Fig. 10 - compatible with its insulating nature - but clearly there are three qualitatively different regimes. At small $\lambda$, the small value of $\delta$ suggests that the charge ordering arises as a consequence of the more dominant zigzag chain formation, as predicted by Hotta et al ${ }^{17}$, namely the spin order dominates over the charge ordering. Increasing $\lambda$, a crossover regime is observed in Fig. 10, which is itself followed by the extreme case of $3+/ 4+$ separation at very large $\lambda$. Since the phase diagram of Fig. 2 shows that 
the FM charge-disordered and CE phases are in contact only below $\lambda=1.2$, this implies an upper bound of approximately $\delta=0.15$ in the charge disproportionation, a result far from the more standard assumption $\delta=0.5$. If $\lambda$ is slightly reduced from 1.2 to $0.8, \delta$ can be as small as 0.05 , compatible with the recent experimental information. As we have stated before, a large Hund coupling suppresses double ocupancy of the same orbital. This interaction together with the electron-phonon interaction and elastic energies behaves like the Hubbard U interaction ${ }^{25}$. The exact equivalence have not yet been clearly established. Our results are in good agreement with van den Brink et al. in the sense that turning on $\lambda$ in our study ( $U$ in Ref 25), the charge migrates from the corner sites of the zigzag chain, to the bridge ones. However, the maximum charge disproportion available in our model $(\delta=0.5)$ differs considerably from the $\delta=0.185$ result obtained for $U=\infty$. These discrepancies deserve further investigations to clarify the range of validity of both models. From all these considerations, our conclusion is that indeed the standard extreme view of the CE state needs revision. In fact, the surprising sensitivity of the CE state to the introduction of disorder (to be discussed below) may be indicative of a fragility which intuitively appears more related to the small- $\lambda$ zigzag-chain-driven regime than to the large $\lambda$ limit. Clearly, the last word on the nature of the CE-state has not been said, and more work should be devoted to this issue.

\section{INFLUENCE OF QUENCHED DISORDER ON THE PHASE DIAGRAM AND FRAGILITY OF THE CE PHASE}

In Fig.11, the numerically obtained phase diagram at fixed $\lambda=1$ varying $J_{\mathrm{AF}}$ is shown in the absence of disorder (cleanlimit). The shape is similar to results presented in previous sections at other $\lambda \mathrm{s}$, with competing FM and CE phases. Shown are also lines of constant spin-spin correlations, which will be useful to understand the results in the presence of quenched disorder. In Fig. 11 , the modified phase diagram after introducing disorder in $J_{\mathrm{AF}}$ is presented. The disorder is included by selecting the coupling $J_{\mathrm{AF}}$ between localized spins at every link as the uniform value plus an extra contribution $\Delta J_{\mathrm{AF}}$, which is randomly added or subtracted (i.e. a bimodal distribution is used). In agreement with the expectations described in the introduction, Fig. 11 including disorder the lines of constant spin correlations bend and reach zero temperature at particular values of $J_{\mathrm{AF}}$. This is to be contrasted with the clean-limit case in Fig. 11], where the lines of constant correlations 'collide' with the CE phase, rather than reaching zero temperature as with disorder incorporated. The behavior of Fig. 11p is indicative of the presence of a spindisordered window separating the FM and CE phases, as in Fig. 11.

However, the situation appears to be more complicated than previous discussions would have suggested. The reason is that the CE phase was here found to be abnormally sensitive to disorder. At least for the value of disorder used, a glassy state is obtained in approximately the same region where the CE phase was found to be stable in the clean limit. The glassy
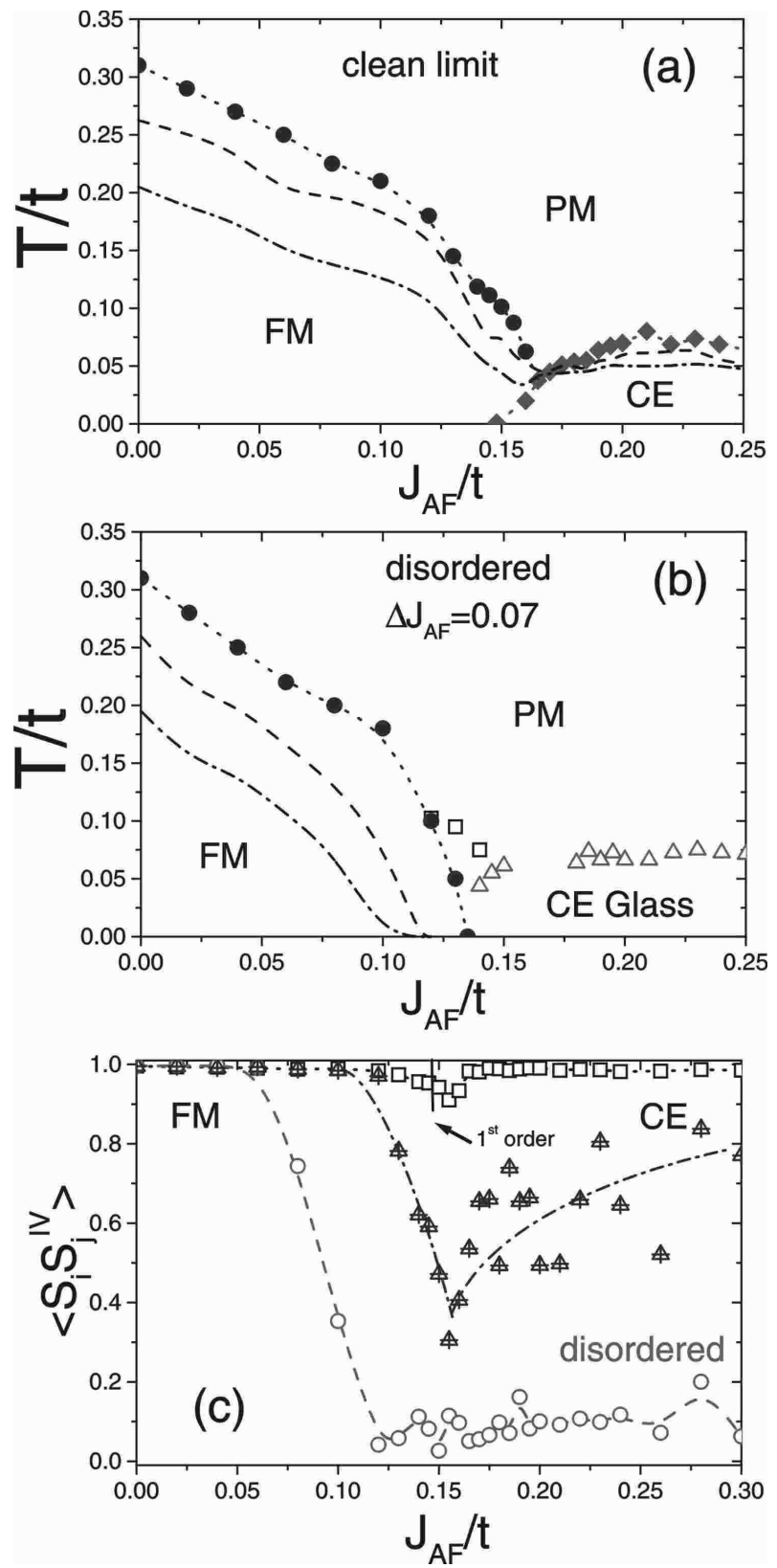

FIG. 11: (a) Phase diagram $T / t$ vs. $J_{\mathrm{AF}} / t$ for the clean limit, with $\lambda=1$. The closed circles and diamonds indicate the transition temperature at which the long-distance correlation $\left\langle S_{i} S_{j}^{I V}\right\rangle$ approximately vanishes upon heating (see text). This is indicative of the temperature where long-range order develops. Dashed and dashdotted lines represent the lines of constant $<S_{i} S_{j}^{I V}>=0.2$, and 0.5 , respectively. (b) Phase diagram $T / t$ vs. $J_{\mathrm{AF}} / t$ for the disorder parameter $\Delta J_{\mathrm{AF}}=0.07$, and $\lambda=1$. The new symbols open -squares and -triangles represent the transition temperatures to a glassy state with short-range correlations but with no obvious long-range FM and CE order, respectively. The CE state was found to be very susceptible to disorder. (c) $<S_{i} S_{j}^{I V}>$ vs. $J_{\mathrm{AF}} / t$ for $\Delta J_{\mathrm{AF}}=0$ (squares), $\Delta J_{\mathrm{AF}}=0.04$ (triangles), and $\Delta J_{\mathrm{AF}}=0.07$ (circles) at low $\mathrm{T}$. These results are the average of five different configurations for the bimodal distribution $J_{i}=J_{\mathrm{AF}} \pm \Delta J_{\mathrm{AF}}$ in the AF links.

nature of the CE region appears in $S(\mathbf{k})$ and it is very clear 
in Monte Carlo snapshots (not shown), that present distorted zigzag chains and patterns of charge ordering - nearly frozen as the Monte Carlo time evolves - in a glassy-looking arrangement. The effect is also manifested in the small value of correlations, as shown in Fig. 11, which contains the spin correlations at the largest distance on the small cluster used. Without disorder, this correlation has the largest allowed value in both the FM and CE phases and as the disorder increases, the correlation reduces its value as expected. What is remarkable is that this effect occurs clearly more rapidly in the $\mathrm{CE}$ region than in the ferromagnetic phase. In fact, at $\Delta J_{\mathrm{AF}}=0.07$ the spin correlations are nearly negligible in the CE regime, indicating the disappearance of order at the largest distances here available. This is to be contrasted against the behavior of correlations in the FM phase, which are far more robust.

The present results are in excellent agreement with the recent experimental studies of $\mathrm{Ln}_{1 / 2} \mathrm{La}_{1 / 2} \mathrm{MnO}_{3}$ ( $\mathrm{Ln}=$ rareearth) by Akahoshi et al $l^{11}$ and Nakajima et al ${ }^{12}$. In these experiments it was observed that the phase diagram has bicritical behavior (Fig. 11 $)$ when the crystals are grown slowly, minimizing by this procedure the influence of disorder. However, upon rapid quenching of the growing process to introduce disorder explicitly, it was observed that while the FM phase reduces its critical temperature by a reasonable amount, the $\mathrm{CE}$ phase is much more affected, turning into a glassy state at low temperatures. This experimentally-observed abnormal sensitivity of the CE phase to disorder -here nicely reproduced in the Monte Carlo simulations- is expected to be an important ingredient for the understanding of the phenomenology of manganites. Disorder indeed plays a key role in the phaseseparated scenario for $\mathrm{Mn}$ oxides $^{2}$, and here its relevance is further confirmed. The simple view expressed in Fig. 1 with a symmetric behavior between the two competing phases, is more complex in practice.

The disorder sensitivity of the CE-phase may arise from the fragility of the zigzag chains. As argued before, the realistic regime of couplings, with $\lambda$ of order 1, presents a chargeordering pattern which is far from the extreme limit $3+/ 4+$, rendering the staggered charge-ordering less robust than previously believed. In addition, the one-dimensional nature of the zigzag chains also contributes to this fragility. Imperfections can easily destroy these nontrivial geometrical arrangement of chains. Unfortunately, the limited size of the clusters used here does not allow us to investigate in more detail the observed CE fragility. These important issues should be analyzed in more detail in future theoretical and experimental investigations. It is interesting to remark that similar conclusions have been recently reached using a one-orbital model with cooperative phonons ${ }^{26}$. Reaching independently the same conclusion using different models, methods, and lattice sizes provides confirmation of the fragility of the chargeordered state that competes with ferromagnetism. This is an important issue that deserves further studies.

\section{TWO TYPES OF CMR}

Let us start the analysis of d.c. transport properties in the half-doped system studied here, aiming toward a better understanding of the CMR effect. In our opinion, these effects should actually be divided in two classes: (1) the CMR effect that occurs at low temperatures and corresponds to very abrupt first-order transitions, and (2) the more standard CMR effect that appears in the regime immediately above the Curie temperature. Years ago, Tokura and collaborators already suggested these two types of $\mathrm{CMRs}^{27}$. Figure 12 provides a direct experimental evidence of this phenomenon, with a huge effect at low temperatures and a relatively smaller effect near $T_{\text {Curie }}$.
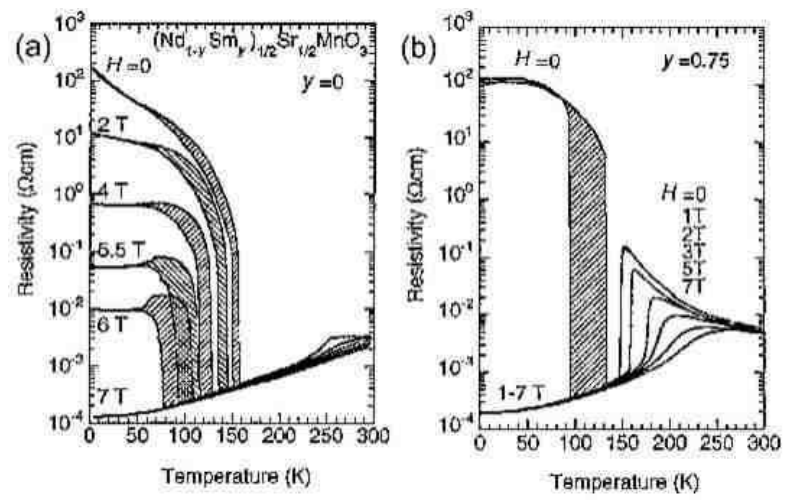

FIG. 12: Temperature dependence of resistivity under various magnetic fields for $\left(\mathrm{Nd}_{1-y} \mathrm{Sm}_{y}\right)_{1 / 2} \mathrm{Sr}_{1 / 2} \mathrm{MnO}_{3}$ with $y=0$ (a) and 0.75 (b). The hatched area represents thermal hysteresis. Results from Tokura et al ${ }^{27}$

This phenomenology - with two CMRs - has recently been discussed by one of the authors (E.D.) in Ref. 2, from where Fig. 13 is reproduced. In this figure, the "CMR1" effect corresponds to a direct field-induced transition from the AF to the FM phases, upon the application of an external field. In this case, quenched disorder is not needed and the transition occurs even in the clean limit. For CMR1 to occur, it is sufficient to be located on the insulating side but close to the first-order transition. Under these circumstances a relatively small field - which obviously favors the FM phase - renders ferromagnetism more favorable and an AF to FM first-order jump is induced. On the other hand, Fig. 13 also shows the standard "CMR2" proccess which is believed to originate in the more complex percolative regime induced by a nanoscale phase separated state above the Curie temperature ${ }^{2.3}$. The realization that there are two types of CMRs substantially clarifies the magnetotransport phenomenology of Mn oxides.

To address explicitly the first type of MR transitions (i.e. CMR1), a clean-limit investigation should be sufficient. To study the resistance of a cluster vs. temperature in the presence of magnetic fields, a setup similar to those often used in investigations of mesoscopic systems will be here employed $^{28}$. This method was recently applied to a technically similar problem of spin and carriers in interaction in the context of diluted magnetic semiconductors 29 . The interacting cluster under investigation is assumed connected to "ideal 


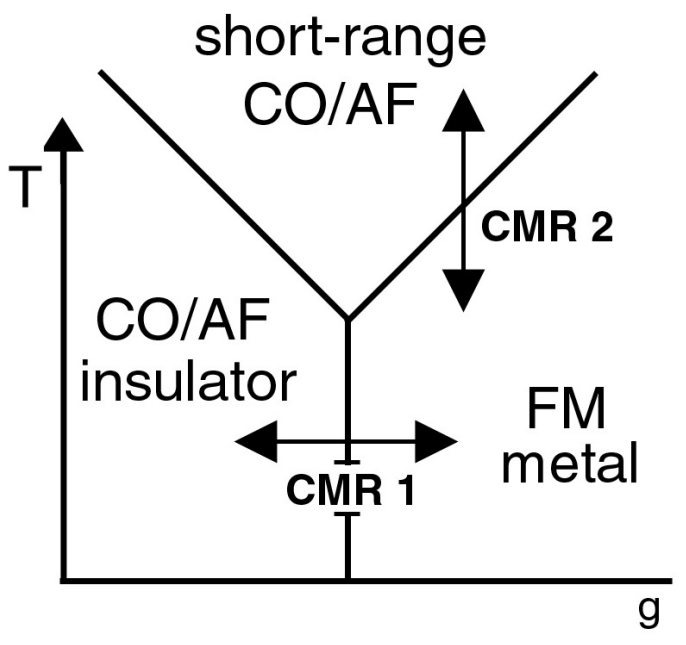

FIG. 13: Schematic representation of the generic phase diagram in the presence of competing FM metal and $\mathrm{CO} / \mathrm{AF}$ insulator, and for quenched disorder not sufficiently strong to destroy entirely the firstorder transition at low temperatures. $g$ is a generic variable needed to transfer the system from one phase to the other. CMR1 and CMR2 are the regions with two types of large MR transitions, as described in the text (see also Ref. 3 )

leads", as shown in Fig. 14 The information about these leads is included through exactly calculated self-energies which are located at the cluster boundaries. The current circulates after an infinitesimal voltage drop is included. The technical aspects have been recently reviewed by Verges 28 and they will not be repeated here. This technique appears to be better than other methods often used in numerical simulations, that rely on the analysis of the Drude weight $D$ of the optical conductivity with the cluster assumed in isolation. In this case, $D$ is often negative due to finite size effects. In addition, the associated zero-frequency delta function must be given an arbitrary width to recover a finite resistivity. These problems are avoided in the present formulation.

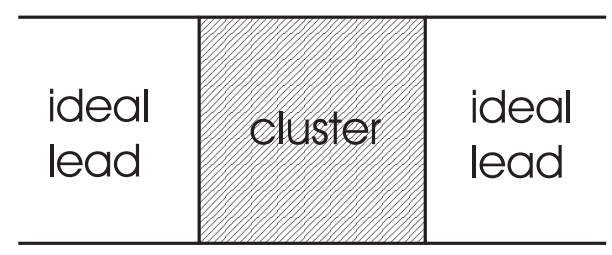

FIG. 14: Geometrical setup used here for the calculation of the resistance of a cluster. For more details see Refs. 2829.

A typical result obtained with the setup of Fig. 14 is shown in Fig. 15. To transform from resistance to resistivity, a cross section of size $4 a \times 1 a$ ( $a=$ lattice spacing) is assumed. In the clean limit, and using as reference the phase diagram of Fig.2. it was observed that for a range of $J_{\mathrm{AF}}$ the material behaves as an insulator since the CE phase dominates, while for other couplings a metallic behavior is found once in the FM regime (here insulator and metal are defined simply based on the sign of the slope of the resistance vs. temperature curves). The technique used here neatly reproduces the expected behavior of the resistivity vs. temperature for a metal and an insulator.
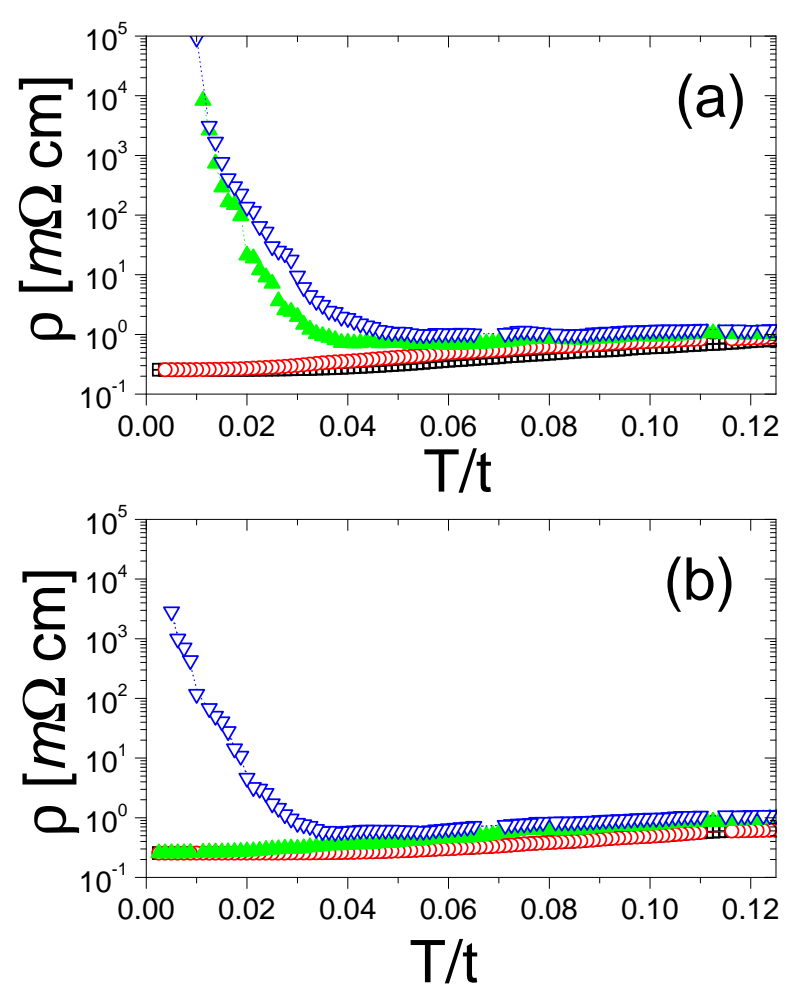

FIG. 15: Clean-limit investigation of the resistivity $\rho$. Shown is $\rho$ vs. temperature, at $\Delta J_{\mathrm{AF}}=0, \lambda=1$, and $J_{\mathrm{AF}}$ equal to 0.14 (squares), 0.16 (circles), 0.18 (closed triangles), and 0.2 (open triangles). The first two $J_{\mathrm{AF}}$ cases correspond to the FM regime, and the other two to the CE state. A first-order transition separates the metal from the insulator at low temperatures, with concomitant abrupt changes in the resistance. (a) corresponds to zero magnetic field $\mu H / t=0$ while (b) is at $\mu H / t=0.05$. Note in the latter that an insulating line in (a), turned metallic in (b), producing a huge MR.

The most important result in this context occurs when magnetic fields are introduced. In this case, the insulating behavior found in the $\mathrm{CE}$ regime close to the FM phase, more precisely at $J_{\mathrm{AF}}=0.18$, turns metallic in a first-order transition upon the application of a field of value $0.05 t$ (much smaller in magnitude than the natural units of the problem, such as $t$ ). Assuming $t$ of the order of $1,000 \mathrm{~K}$, this is a field of about $50 \mathrm{~T}$, larger but not by a huge amount with respect to those typically used in CMR experiments. By selecting $J_{\mathrm{AF}}$ even closer to the first-order transition the value of the field needed to induce metallic behavior can be easily reduced. The associated MR ratios - defined as $\mathrm{MR}=(\rho(0)-\rho(H)) / \rho(H) \times 100 \%$, with $H$ the field used - are shown in Fig. 16. For the coupling where the insulator-metal transition was generated by the magnetic field, the MR ratio was found to be as large as $10^{7 \%}$ at low temperatures, in excellent agreement with the values that can be deduced from the experimental data in Fig. 12 As explained before, this drastic effect is simply caused by a level crossing induced by the magnetic field, which favors the FM phase. Note that the $J_{\mathrm{AF}}$ coupling used is not abnormally 
close to the original transition, namely there is no need to carefully fix parameters to see this effect. The present calculation clearly shows that huge MR effects can be obtained in theoretical calculations, even in small clusters and without the need of tuning couplings.
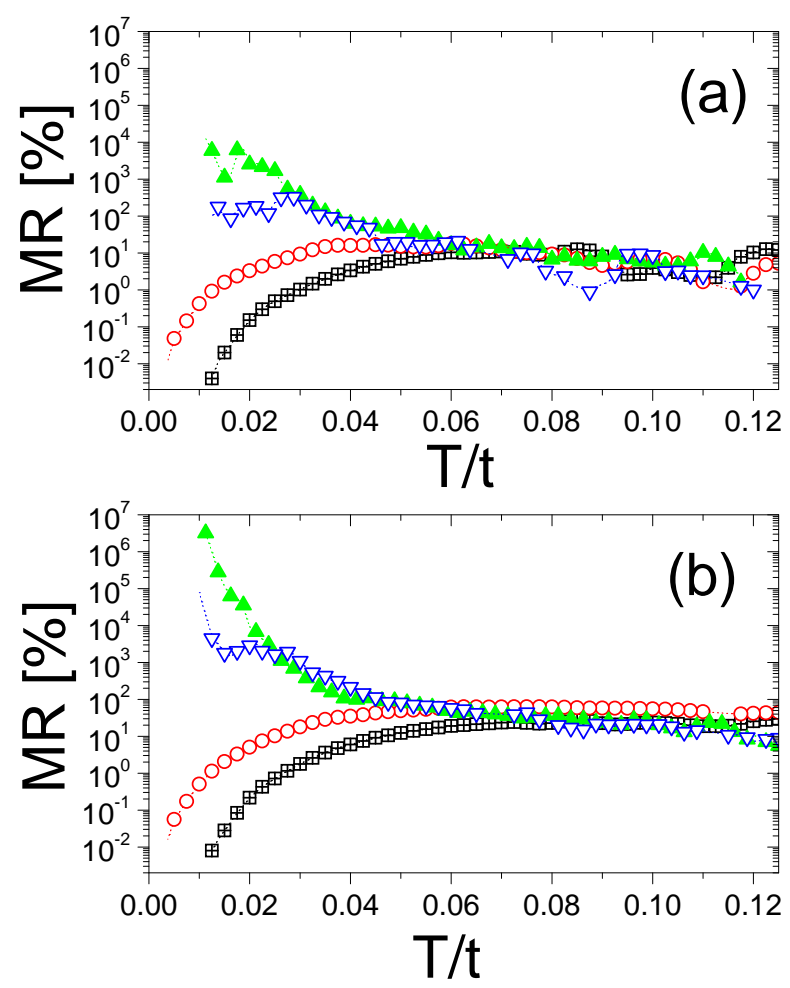

FIG. 16: Clean-limit magnetoresistance (as defined in text) vs. temperature considering $\mu H / t=0.025$ (a), and $\mu H / t=0.05$ (b). The same set of parameters and convention of symbols are followed as in Fig. 15 Huge MR ratios are observed, as found in experiments.

Regarding the theoretical understanding of the more standard CMR (namely, CMR2 in the present discussion), previous investigations have relied on sophisticated calculations using simplified models and resistor networks in order to capture the complex percolative physics expected to dominate in real compounds $\mathrm{s}^{2,3}$. These subtle effects cannot be studied in the small clusters currently accessible to nearly-exact Monte Carlo studies of realistic models, as those presented here. However, results in those small clusters when quenched disorder is included, already provide hints of the physics found in experiments and in simulations of toy models. For example, in Fig. 17 it is shown that the disorder reduces the resistivity in the CE regime, and that this effect is further magnified when magnetic fields are applied. Unfortunately, in spite of observing these reasonable effects, the shape of the resistivity curves still do not show the well-known metal-insulator transition at a finite temperature. Further computational work will be needed to reproduce the CMR2 effect using realistic Hamiltonians. Fortunately, the essence of the phenomenon appears to have been captured by the calculations of Ref. 3 .
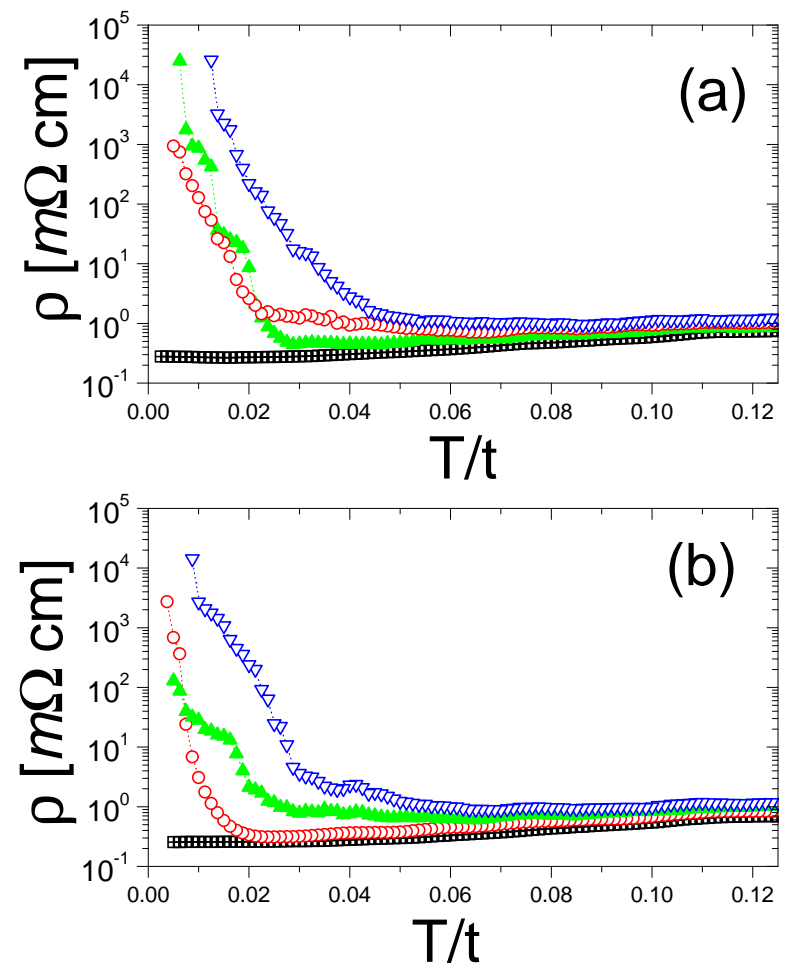

FIG. 17: Study of the resistivity $\rho$ in the presence of quenched disorder. Shown is $\rho$ vs. temperature, for $\mu H / t=0$ (a), and $\mu H / t=0.025$ (b), using $\lambda=1, \Delta J_{\mathrm{AF}}=0.04$, and $J_{\mathrm{AF}}$ equal to 0 (squares), 0.04 (circles), 0.08 (closed triangles), and 0.12 (open triangles). Large MR effects are observed, even involving only insulating phases.

\section{CHARGE-ORDERING IN ELECTRON-DOPED MATERIALS}

For completeness, here results at electronic densities $\langle n\rangle$ different from 0.5 are also presented. However, the 'halfdoped' character of the investigation remains. To be more specific, the emphasis in this section is on $\langle n\rangle=1.5$, namely in the regime of electron doping of undoped compounds such as $\mathrm{LaMnO}_{3}$, opposite to the hole-doping regime of $\langle n\rangle=0.5$ discussed in the rest of the paper. The experimental motivation for this effort relies on recent investigations that have reported results for $\mathrm{La}_{0.7} \mathrm{Ce}_{0.3} \mathrm{MnO}_{3}$, which is indeed an electrondoped compound having $\mathrm{LaMnO}_{3}$ as the parent materiall3. A ferromagnetic metallic phase has been reported in this context with a Curie temperature $\sim 250 \mathrm{~K}$ and a large magnetoresistance, establishing clear similarities with the well-known results for the hole-doped region of the phase diagram.

These experiments suggest that there must be a relation between electron and hole doping, which has not been discussed theoretically before to our knowledge. In particular, it is interesting to speculate what kind of states will be obtained in the electron-doped regime once the doping is made as large as $50 \%$ (i.e. $\langle n\rangle=1.5$ ). In other words, here the study will focus on the discussion of what kind of $\mathrm{CO} / \mathrm{OO}$ state could be obtained in the electron half-doped regime of manganites, 
justifying the inclusion of the results described below in the present paper, which is devoted to half-doped manganites in general.

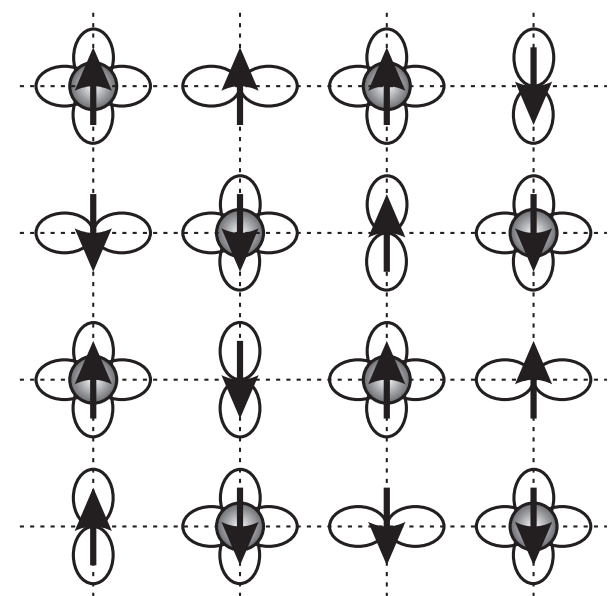

FIG. 18: Orbital, spin, and charge arrangement for the electron overdoped $(\langle n\rangle=1.5) \mathrm{CE} / \mathrm{CO} / \mathrm{OO}$ phase. The $3 x^{2}-r^{2}$ and $3 y^{2}-r^{2}$ orbitals represent the $\mathrm{Mn}^{3+}$ ions. The superposition of the $x^{2}-y^{2}$ plus $3 z^{2}-r^{2}$ orbitals (closed circles) represents the $\mathrm{Mn}^{2+}$ ions.

The main conclusion of the analysis of the $\langle n\rangle=1.5$ phase diagram using Monte Carlo simulations is that in the large Hund coupling limit, there is a mapping between $\langle n\rangle$ bigger and smaller than one (with $\langle n\rangle=1$ corresponding to $\mathrm{LaMnO}_{3}$ ). In fact, in the $J_{\mathrm{H}}=\infty$ limit studied here the phase diagrams in the plane $\lambda-J_{\mathrm{AF}}$ are identical (within numerical accuracy) with regards to the location of the phase boundaries. However, the characteristics of each phase cannot be the same in view of the different electronic densities. As particular case, our effort predicts that the analog of the well-known CE phase of half hole-doped manganites is the state shown schematically in Fig. 18 In this state, there is a separation of charge into a ' $3+/ 2+$ ' like configuration, to be contrasted with the standard $3+/ 4+$ state at $\langle n\rangle=0.5$. The zigzag chains of the standard CEstate are still present, and even the orbitals in the middle of the segments of the zigzag chains are elongated along the $x$ and $y$ axis. The main difference between $\langle n\rangle=0.5$ and 1.5 is the state at the vertices of those chains, which is empty at density 0.5 but it contains two electrons at 1.5 . An analog situation is found in the other charge-ordered phases. It would be very interesting to confirm experimentally whether this state is present in real half-electron-doped manganites. Note that our calculation does not include the effect of Coulombic interactions, which may be more important in the electron-doping regime than in the hole-doping region. However, in other materials such as the cuprates, a strong similarity between electron- and hole-doping has been found as well, suggesting that a similar situation may occur in manganites. Completing the phase diagram of electron-doped manganites would help us in achieving a deeper understanding of these materials. The concrete prediction of our efforts is that there should be an approximate particle-hole symmetry with respect to the undoped $\mathrm{LaMnO}_{3}$ compound, in a similar spirit as it occurs in the cuprates ${ }^{30}$.

\section{CONCLUSIONS}

The investigations reported in this paper have unveiled several unexpected properties of half-doped manganites. For example, the CE phase was found to be more sensitive than expected to the addition of quenched disorder. The effort in this context included the calculation of the clean-limit phase diagram in the $T-J_{\mathrm{AF}}$ plane, to understand the competition between the FM and CE states believed by most manganite experts to be at the heart of the CMR phenomenon. In agreement with previous calculations ${ }^{2.3}$, a low-temperature firstorder transition between the two phases was found, with a shape similar to those reported experimentally ${ }^{9}$. Adding disorder, the predicted ${ }^{3}$ depletion of critical temperatures was observed, but contrary to those expectations the reduction was not symmetrical in magnitude for the two competing phases. In fact, it was observed that the CE state rapidly transforms into a "CE glass" with disorder, while the ferromagnetic phase is comparatively less affected, in nice agreement with recent experiments where disorder is introduced "by hand" upon a clean-limit bicritical-shaped phase diagram ${ }^{11.12}$. The zigzag chains of the CE state and its very subtle arrangement of spins, charge, and orbitals seems easily broken by imperfections, contrary to the more robust uniform FM state.

The present results revealed other new properties of manganite states that also deserve further investigations. Among these interesting new properties are the existence of novel phases, such as a FM/CO state, and a discussion on charge disproportionation that suggests that the widely held view of charge-ordering as containing $3+$ and $4+$ ions needs considerable revision.

A second important result presented here is the existence of a very large MR effect when the insulating state is close in energy to the FM state, as it occurs in the first-order transitions systematically found in the clean limit. As a consequence, without quenched disorder and working at low temperatures, the insulating state can be destabilized by the FM state with increasing fields. The magnitude of the field required can be very small in units of the hopping, and its actual critical value depends on how close the CE state investigated is to the FM state in the phase diagram (i.e. how close the analyzed value of $J_{\mathrm{AF}}$ is to the critical value separating the phases). This MR effect is qualitatively different from the more standard effect found above the Curie temperature, which is believed to need disorder effects to be understood ${ }^{2,3}$. As predicted by Tokura and collaborators 27 many years ago, there are two types of CMR: a low-temperature form - investigated theoretically here - and a higher temperature variety with the standard profiles for resistivity vs. temperature. In carrying out these investigations, we have introduced techniques borrowed from mesoscopic physics, within the context of the Landauer formalism.

The study of electron-doped systems has also been initiated in the investigations reported in this paper, focusing on the half-doped limit. Charge-ordered states analogous to those observed at half-hole-doping were identified. A formal particle-hole symmetry appears to exist in the system with respect to the undoped $\mathrm{LaMnO}_{3}$ limit, at least for a large Hund 
coupling. More theoretical work in this area should be pursued, since experiments are starting to investigate electrondoped manganites.

It is clear that the study of models for manganites using unbiased techniques provides a plethora of interesting results. In the field of manganites the crossfertilization theoryexperiments has been remarkably positive, and new surprises will likely be found in the near future. Investigations of manganites and other related oxides should continue at its present rapid rate, to enhance our understanding of correlated electron systems in general.

\section{ACKNOWLEDGMENTS}

H. A. is supported by the Schuler Fellowship at the Magnet Lab. A. M. and E. D. are supported by the NSF grant DMR0122523. Additional funds have been provided by Martech (FSU). We acknowledge the help of G. Alvarez and J. Verges in the study of the resistance of clusters reported here and useful conversations with T. Hotta and L. Brey. The School of Computational Science and Information Technology (CSIT) at FSU is also acknowledged.

\section{APPENDIX: SIMULATIONS IN THE CANONICAL ENSEMBLE}

In most of our simulations, we have kept the number of particles fixed in our systems basically at each Monte Carlo
Step per Site (MCS/S). In order to achieve that, we have to solve the equation,

$$
n-\sum_{i=1}^{N} \sum_{k=1}^{2 N} \frac{c_{k, i}^{+} c_{k, i}}{1+\exp \left[\left(E_{k}-\mu\right) / T\right]}=0
$$

for the chemical potential, $\mu$; where $n$ is the desired number of electrons in the system, $N$ is the number of sites of the cluster, $c_{k, i}^{+}$and $c_{k, i}$ are the fermionic operators after diagonalizing the hamiltonian matrix (Eq. (1)), and $E_{k}$ are the electronic energy levels. These energies are ordered in the way: $E_{2 N} \leq E_{k} \leq$ $E_{1}$, with $k$ running between 1 and $2 N$. The first sum symbol runs over sites, while the second runs over energy levels $(2 \mathrm{~N}$ in our case, since we have two orbitals per site).

We have solved this equation using the Newton-Raphson method $^{31}$, starting with an initial seed $\mu_{0}=1 / 2\left(E_{2 N-l}+\right.$ $\left.E_{2 N-l+1}\right)$, with $l=\operatorname{int}(x N)$, and the filling $x$ given by: $x=n / N$. The symbol int( ) means the nearest integer of a real number. Fixing an absolute error for $n$ of $10^{-5}$, the chemical potential $\mu$ is typically found in 4-5 iterations. At sufficiently low temperatures, $k_{\mathrm{B}} T / t \leq 0.01$ for example, the convergence of this method can fail. In this case we use the auxiliary bisection method ${ }^{31}$, where typically $20-30$ steps are needed for the same accuracy.
1 A. Moreo, S. Yunoki and E. Dagotto, Science 283, 2034 (1999); E. Dagotto, T. Hotta, and A. Moreo, Phys. Reports 344, 1 (2001); A. Ramirez, J. Phys.: Cond. Matt. 9, 8171 (1997); J. Coey, M. Viret, and S. von Molnar, Adv. Phys. 48, 167 (1999); Y. Tokura and Y. Tomioka, J. of Mag. and Mag. Materials 200, 1 (1999); M. R. Ibarra and J. M. De Teresa, Materials Science Forum Vols. 302303, 125 (1999); Trans Tech Publications, Switzerland; C. Rao et al., J. Phys.: Cond. Matt. 12, R83 (2000); A. Moreo, Journal of Electron Spectroscopy and Related Phenomena 117-118, 251 (2001); M. B. Salamon and M. Jaime, Rev. Mod. Phys. 73, 583 (2001); M. Imada, A. Fujimori, and Y. Tokura, Rev. Mod. Phys. 70, 1039 (1998); E. Dagotto, Rev. Mod. Phys. 66, 763 (1994); C. N. R. Rao and B. Raveau, editors, Colossal Magnetoresistance, Charge Ordering, and Related Properties of Manganese Oxides, World Scientific, Singapore, 1998; T. A. Kaplan and S. D. Mahanti, editors, Physics of Manganites, Kluwer Academic/Plenum Publishers, New York, 1998; Y. Tokura, editor, Colossal Magnetoresistive Oxides, Gordon and Breach, 2000; and many others.

2 E. Dagotto,Nanoscale Phase Separation and Colossal Magnetoresistance, Springer-Verlag, Berlin, 2002.

3 J. Burgy, M. Mayr, V. Martin-Mayor, A. Moreo, E. Dagotto, Phys. Rev. Lett. 87, 277202 (2001).

${ }^{4}$ M. Uehara, S. Mori, C. H. Chen, and S.-W. Cheong, Nature 399, 560 (1999); M. Fäth, S. Freisem, A. A. Menovsky, Y. Tomioka, J. Aarts, and J. A. Mydosh, Science 285, 1540 (1999).

5 S. H. Pan, J. P. O’Neal, R. L. Badzey, C. Chamon, H. Ding, J. R. Engelbrecht, Z. Wang, H. Eisaki, S. Uchida, A. K. Gupta, K-W.
Ng, E. W. Hudson, K. M. Lang, and J. C. Davis, Nature 413, 282 (2001).

${ }^{6}$ E. Dagotto, Open Questions in CMR Manganites, Relevance of Clustered States, and Analogies with Other Compounds, cond-mat/0302550

7 T. Hotta, A. Feiguin, and E. Dagotto, Phys. Rev. Lett. 86, 4922 (2001).

8 S. Yunoki, T. Hotta, and E. Dagotto, Phys. Rev. Lett. 84, 3714 (2000).

9 Y. Tomioka, and Y. Tokura, Phys. Rev. B. 66, 104416 (2002); and references therein.

10 J. C. Loudon, N. D. Mathur, and P. A. Midgley, Nature 420, 797 (2002).

11 D. Akahoshi, M. Uchida, Y. Tomioka, T. Arima, Y. Matsui, and Y. Tokura, Phys. Rev. Lett. 90, 177203 (2003).

12 T. Nakajima, H. Kageyama, and Y. Ueda, J. Phys. Chem. 63, 913 (2002).

13 P. Mandal and S. Das, Phys. Rev. B 56, 15073 (1997); C. Mitra, P. Raychaudhuri, J. John, S. K. Dhar, A. K. Nigam, and R. Pinto, J. Appl. Phys. 89, 524 (2001); C. Mitra, Z. Hu, P. Raychaudhuri, S. Wirth, S. I. Csiszar, H. H. Hsieh, H.-J. Lin, C. T. Chen, and L. H. Tjeng, cond-mat/0206137, Phys. Rev. B 67, 092404 (2003); and references therein.

14 T. Hotta, S. Yunoki, M. Mayr, and E. Dagotto, Phys. Rev. B 60, R15009-R15012 (1999); M. N. Iliev, M. V. Abrashev, H.-G. Lee, V. N. Popov, Y. Y. Sun, C. Thomsen, R. L. Meng, and C. W. Chu, Phys. Rev. B 57, 2872 (1998). 
15 In practice, considering sites $\mathbf{i}$ and $\mathbf{i}+\mathbf{a}$, the oxygen in between is allowed to move along the a-axis, i.e., buckling and rotations are neglected. In the $2 \mathrm{D}$ case apical oxygens are assumed to be fixed, consistent with previous treatments to produce stripe-like charge ordering in the 2D FM-phase ${ }^{7}$. Note that the apical oxygens freezing makes the $Q_{1}$ and $Q_{3}$ modes equivalent and their relative importance can not be disentangled. In our studies $Q_{2}$ is typically the most important mode.

16 S. Yunoki, A. Moreo, and E. Dagotto, Phys. Rev. Lett. 81, 5612 (1998).

17 T. Hotta, Y. Takada, H. Koizumi, and E. Dagotto, Phys. Rev. Lett. 84, 2477 (2000).

18 Takashi Hotta, Mohammad Moraghebi, Adrian Feiguin, Adriana Moreo, Seiji Yunoki, and Elbio Dagotto, Phys. Rev. Lett. 90, 247203 (2003).

19 T. Kimura, S. Ishihara, K. T. Takahashi, H. Shintani, and Y. Tokura, cond-mat/0211568 See also A. Muñoz et al., Inorg. Chem. 40, 1020 (2001).

20 A. Moreo, S. Yunoki, and E. Dagotto, Phys. Rev. Lett. 83, 2773 (1999).

${ }^{21}$ D. S. Dessau, T. Saitoh, C.-H. Park, Z.-X. Shen, P. Villella, N. Hamada, Y. Moritomo, and Y. Tokura, Phys. Rev. Lett. 81, 192 (1998).

${ }^{22}$ K. H. Kim, S. Lee, T. W. Noh, and S.-W. Cheong, Phys. Rev. Lett. 88, 167204 (2002).

${ }^{23}$ E. Dagotto, J. Burgy and A. Moreo, Nanoscale Phase Separation in Colossal Magnetoresistance Materials: Lessons for the Cuprates?, Solid State Communications 126 , 9 (2003), cond-mat/0209689 Proceedings of the post- $\mu$ SR2002 Superconductivity Workshop, Williamsburg, VA., June 3-7, 2002, edited by A.J. Millis, S. Uchida, Y.J. Uemura.

24 J. Garcia, M. C. Sanchez, G. Subias, and J. Blasco, J. Phys.:Condens. Matter 13, 3229 (2001); J. Garcia, M. C. Sanchez,
J. Blasco, G. Subias, and M. G. Proietti, J. Phys.:Condens. Matter 13, 3243 (2001); A. Daoud-Aladine, J. Rodriguez-Carvajal, L. Pinsard-Gaudart, M. T. Fernandez-Diaz, and A. Revcolevschi, Phys. Rev. Lett. 89, 097205. See also M. C. Sanchez, G. Subias, J. Garcia, and J. Blasco, Phys. Rev. Lett. 90, 045503 (2003); Comment on "X-ray resonant scattering studies of orbital and charge ordering in $\operatorname{Pr}_{1-x} \mathrm{Ca}_{x} \mathrm{MnO}_{3}$ " by J. Garcia and G. Subias; G. Zheng and C. H. Patterson, cond-mat/0302622 V. Ferrari and P. B. Littlewood, "Oxygen Stripes in $\mathrm{La}_{0.5} \mathrm{Ca}_{0.5} \mathrm{MnO}_{3}$ from ab initio calculations", preprint.

25 J. van den Brink, G. Khaliullin, and D. Khomskii, Phys. Rev. Lett. 83, 5118 (1999).

26 Y. Motome, N. Furukawa, and N. Nagaosa, private communication.

27 Y. Tokura, H. Kuwahara, Y. Moritomo, Y. Tomioka, and A. Asamitsu, Phys. Rev. Lett. 76, 3184 (1996).

28 J. A. Verges, Computer Physics Communications 118, 71 (1999); and references therein.

${ }^{29}$ G. Alvarez and E. Dagotto, Single-Band Model for Diluted Magnetic Semiconductors: Dynamical and Transport Properties, and Relevance of Clustered States, submitted to PRB.

${ }^{30}$ In fact, the usual particle-hole transformation $d_{\mathbf{i} \gamma}^{\dagger} \rightarrow c_{\mathbf{i} \gamma}(-1)^{i_{x}+i_{y}}$, with $d$ the fermionic operator at infinite Hund coupling and $\mathbf{i}=\left(i_{x}, i_{y}\right)$, supplemented by a change of sign from $u$ to $-u$ in all the phononic variables, can be shown to be a symmetry of the Hamiltonian. Whether this symmetry is or not of relevance in regimes of spontaneous-symmetry-breaking, namely when not necessarily both $u$ and $-u$ contribute to the partition function equally, needs further studies.

31 W. H. Press, S. A. Teukolski, W. T. Vetterling, and B. P. Flannery, Numerical Recipes, Cambridge University Press, 1992. 